\title{
HERNIATION THROUGH LESSER SAC: A RARE TYPE OF PRIMARY INTERNAL HERNIA CAUSING SMALL INTESTINAL OBSTRUCTION
}

\section{General Surgery}

Dr. Pritam Kar

Junior Resident Academic, Department of General Surgery ,Rajendra Institute of Medical Sciences, Ranchi ,Jharkkhand 834009.

Dr. Ranjan George Professor and Head, Department of General Surgery ,Rajendra Institute of Medical Baxla

\section{Dr. Abhishek} Kushwaha* Sciences, Ranchi, Jharkkhand 834009.

Dr. Anup Kumar Mahato

Junior Resident Academic, Department of General Surgery ,Rajendra Institute of Medical Sciences, Ranchi, Jharkkhand 834009.*Corresponding Author

Junior Resident Academic, Department of General Surgery ,Rajendra Institute of Medical Sciences, Ranchi ,Jharkkhand 834009.

\section{ABSTRACT}

Primary internal hernia is a rare phenomenon, where there is protrusion of an abdominal organ mostly gut through epiploic foramen. Clinical presentation of internal hernia is non specific.Imaging has been of limited utility in cases of acute intestinal obstruction; moreover, interpretation of imaging features is operator dependant. Thus internal hernias are usually detected at laparotomy and preoperative diagnosis in an emergency setting is either difficult or most of the time not suspected We describe the case of a 14-year-old male who presented with acute abdominal pain and abdominal distension with no history of trauma .A loop of jejunum was found to enter the lesser sac with multiple peforated jejunal loop $35 \mathrm{~cm}$ distal to dj junction. .The segment of perforated bowel loop of length $10 \mathrm{~cm}$ was resected and jejuno-jejunostomy was done.This is a rare type internal hernia into lesser sac.

\section{KEYWORDS}

Primary internal hernia ; congenital internal abdominal hernia; lesser sac herniation

\section{INTRODUCTION}

An internal hernia is a protrusion of intestines or other abdominal organs through a normal or abnormal orifice in the peritoneum or mesentery, which may lead to strangulation. Internal hernias(IH) rarely cause acute abdomen and intestinal obstruction with incidence rate of 0.2 to $0.9 \%$ and 0.6 to $5.8 \%$ of the cases[1,2]. Preoperative diagnosis in an emergency setting are difficult due to rarity of the entity, nonspecific clinical presentation, and limited utility of imaging in cases of acute intestinal obstruction [3].A 14-yr old male presented in our casualty with acute intestinal obstruction.

\section{Case Presentation}

A 14-year-old male was admitted in emergency with complaints of acute abdominal pain with distension and inability to pass stool and flatus for five days.. His symptoms aggravated with increased episodes of bilious vomiting. He had no histroy of any fever, dysuria, hematochezia, past surgical intervention, or trauma

On examination ,pulse rate was 104/minute and blood pressure was $100 / 80 \mathrm{~mm}$ of $\mathrm{Hg}$. Patient was afebrile and tachypneic. Abdominal distension and tenderness were detected on abdominal examination. Bowel sounds were absent. Digital rectal examination revealed rectum empty with ballooning of rectum.

$\mathrm{X}$ ray abdomen(erect) showed dilated small intestinal loops with multiple air-fluid levels suggesting intestinal obstruction. Ultrasonography shows distended bowel loops .CT scan was done. Routine blood reports were within normal limits.

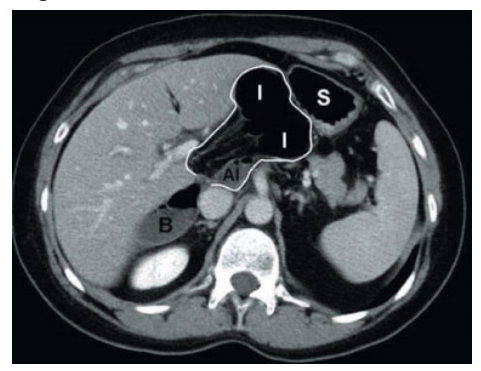

FIGURE 1:CT showing small bowel loops(I) located within the lesser sac (white line) through foramen of Winslow between liver and inferior vena cava with a narrowed afferent loop(AI) passing through foramen of Winslow .The stomach (S) is displaced anteriorly and laterally and the bowel loops (B) show dilation proximal to afferent loops.

Surgeons performed exploratory laparotomy .0n laparotomy, omentum was found covered to small intestine adherent to each other. Adhesionolysis was done.

$\mathrm{X}$ ray abdomen(erect) showed dilated small intestinal loops with multiple air-fluid levels suggesting intestinal obstruction.

Ultrasonography

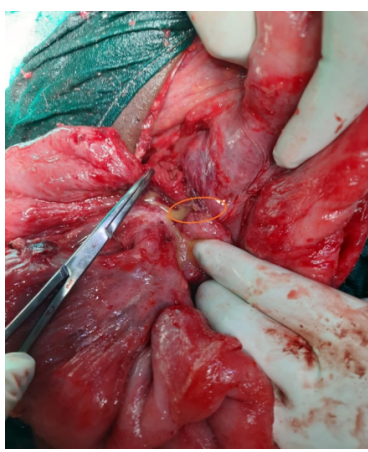

FIGURE 2:Herniated bowel loop entering through foramen of winslow(marked with circle) with pus draining from lesser sac and through and through jejunal rent $35 \mathrm{~cm}$ from dj junction (pointed by artery forceps)

Well defined sac of size $15 \mathrm{~cm} * 10 \mathrm{~cm} * 10 \mathrm{~cm}$ was identified shifting stomach upward and transverse colon downwards;identified to be lesser sac that sac was filled with pus which was drained and a loop of jejunum was found to be herniated into it. Two small rents and one large through and through rent of size $2 \mathrm{~cm} * 2 \mathrm{~cm}$ was present $35 \mathrm{~cm}$ distal to duodeno-jejunal junction. About $10 \mathrm{~cm}$ of jejunum with multiple rents was resected and jejuno-jejunostomy done. The sac was closed with nonabsorbable interrupted suturing. The patient made a slow recovery and was discharged 2 weeks later.

\section{DISCUSSION}

Internal hernias are of two types: congenital and acquired, the latter constituting the majority. Acquired internal herniations are caused by previous abdominal surgery, trauma[4].Primary or Congenital internal 
abdominal hernias (CIAH) are mostly retroperitoneal in origin or formed from congenital anomalous openings that lacks a true peritoneal sac. Ghahremani classified retroperitoneal hernias into paraduodenal (30-53\% of CIAH), foramen of Winslow(6-10\%), pericecal $(10-15 \%)$, intersigmoid $(4-8 \%)$, pelvic,and paravesical hernias $(6 \%)$. CIAHs can be categorized as transmesenteric (8-10\%), broad ligament (4-7\%), or transomental hernias (1-4\%) [5].

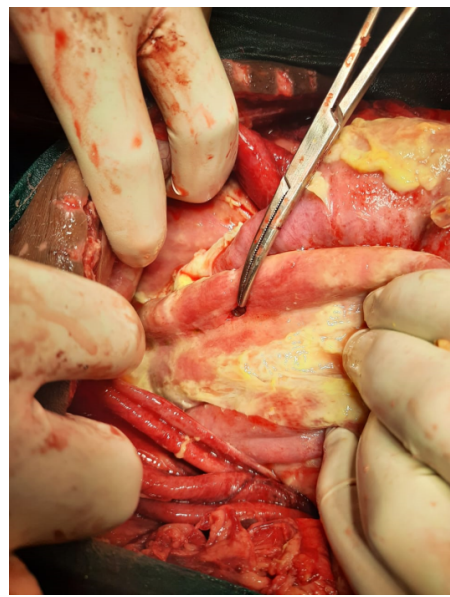

FIGURE 3:Lesser sac (wall retracted by finger) with perforated jejunum (pointed by artery forceps) due to strangulation of small bowel into lesser sac with flakes of debris.

In this case, a lesser sac hernia of gut was present herniating through foramen of winslow. It usually presents with ischemia and late cases of perforation[7].Symptom severity relates to duration and reducibility of the hernia and the presence or absence of strangulation [8].Clinical presentation is nonspecific posing a diagnostic challenge preoperatively eventually leading to diagnostic delays and the resultant increase in rates of ischemia, gangrene and bowel perforation as shown below.

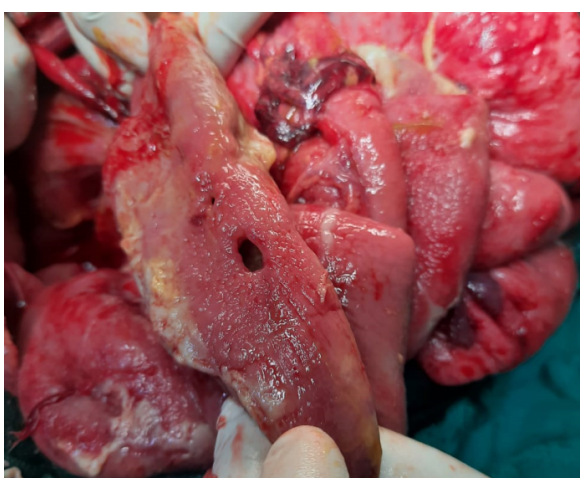

FIGURE 4:Jejunal rent with blunt edges signifying old nature of perforation due to diagnostic delays due to nonspecific symptoms of $\mathrm{SiO}$.

Radiographs and ultrasonography (23\% detection) are poor in detecting the cause of intestinal obstruction.CT scan being diagnostic identifies specific site, severity, closed loop and multiple segments of obstruction[9]. CT features depicts mass or cluster of dilated small bowel loops at abnormal anatomical location with $\mathrm{SiO}$ and with engorged, stretched ,or displaced mesenteric vascular pedicle and of converging vessels at the hernia orifice [10]. CT can detect ischemia, necrosis, or perforation and inflammatory changes[11]. However, the $\mathrm{CT}$ diagnosis of $\mathrm{IH}$ remains difficult even after its accessibility and use [9].Reduction of the strangulated intestinal segment to be done to prevent intestinal ischemia ,necrosis , and perforation;reducing resection rates [12]. Hernia defects are usually closed with nonabsorbable sutures thus preventing recurrence.

\section{CONCLUSION}

In acute abdomen though it is important to make an accurate preoperative diagnosis, it is far more important for detailed assessment of the patient's symptoms and signs and decision making about surgical intervention to avoid detrimental complications like the bowel perforation, diagnosis of peritonitis being delayed, which compromises outcome due to septicemia. In this particular case diagnosis of intestinal obstruction was delayed so much so that patient developed jejunal perforations. Naturally it must have increased morbidity in terms of wound and abdominal sepsis. Internal hernias are rare entity and in every case accurate preoperative diagnosis is not expected however a timely surgical intervention (Early laparotomy) is needed for whenever patient presents with symptoms and signs of acute intestinal obstruction due to any cause.

\section{REFERENCES}

[1] E.-J. Jang S. H. Cho, and D.-D. Kim, A case of small bowel obstruction due to paracecal hernia, Journal of the Korean Society of Coloproctology,vol.27,no.1,pp.4143,2011

[2] A. Rae, A. Kalyanaraman, A. Ward, and A. Harikrishnan, An interesting case of retro caecal internal herniation causing small bowel obstruction, Annals of Medicine and Surgery, 2015

[3] S. Ghiassi, S. Q. Nguyen, C. M. Divino, J. C. Byrn, and A. Schlager, Internal hernias clinical findings ,management, and outcomes in 49 non bariatric cases, Journal of Gastrointestinal Surgery,vol.11,no.3,pp.291-295,2007.

[4] H.M.Edwards and H.Al-Tayar, A trans mesenteric congenital internal hernia presenting in an adult, Journal of Surgical Case Reports, vol. 2013, no. 12, article rjt $099,2013$.

[5] M.Takeda ,Y.Ohnuki ,T.Uchiyama ,O.Kubota, and K.Ohishi, Small intestinal strangulation due to a rare type of primary internal hernia, International Surgery, vol. 98 , no. 4, pp. 409-411,2013

[6] T.Nonaka, Y.Shibata,K.Tou,S.Honjyou, and T.Oka, A case of retroperitoneal hernia in paracolic gutter of the descending colon, Japanese Journal of Gastroenterological Surgery, vol.41 no.6,pp.700-704,2008

[7] M. Erikoglu and M. A. Yildirim, Internal herniation due to appendix vermiformis rotation, European Journal of General Medicine,vol.9, no.4,pp.295-297,2012.

[8] Y. Narjis and B. Finech, Primitive internal hernia in adult: a report on 6cases, Surgery:Current Research,vol.5, article235, 2015.

[9] L. C. Martin, E. M. Merkle, and W. M. Thompson, Review of internal hernia radiographic and clinical findings , American Journal of Roentgenology, vol. 186, no.3,pp.703-717,2006

[10] N.Takeyama, T.Gokan, Y.Ohgiya et al., CT of internal hernias, Radiographics, vol.25,no.4,pp.997-1015,2005.

[11] F. Lassandro, F. Iasiello, N. L. Pizza et al., Abdominal hernias: radiological features, World Journal of Gastrointestinal Endoscopy,vol.3,no.6,pp.110-117,2011.

[12] R.Gomes and J.Rodrigues, Spontaneous adult transmesentric hernia with bowe gangrene, Hernia,vol.15,no.3,pp.343-345, 2011. 